\title{
NEW EXISTENCE RESULTS ON NONHOMOGENEOUS STURM-LIOUVILLE TYPE BVPS FOR HIGHER-ORDER $p$-LAPLACIAN DIFFERENTIAL EQUATIONS
}

Abstract. A class of nonlinear boundary value problems for $p$-Laplacian differential equations is studied. Sufficient conditions for the existence of solutions are established. The nonlinearities are allowed to be superlinear. We do not apply the Green's functions of the relevant problem and the methods of obtaining a priori bounds for solutions are different from known ones. Examples that cannot be covered by known results are given to illustrate our theorems.

1. Introduction. In [3, 4, Erbe and Tang studied the Sturm-Liouville boundary value problem (BVP for short) for the second order differential equation which comes from the situation involving nonlinear elliptic problems in annular regions.

Recently, Qi [8] investigated the following BVP for a higher-order differential equation:

$$
\left\{\begin{array}{l}
x^{(n)}(t)+f\left(t, x(t), x^{\prime}(t), \ldots, x^{(n-2)}(t)\right)=0, \quad 0<t<1, \\
x^{(i)}(0)=0 \quad \text { for } i=0,1, \ldots, n-3, \\
\alpha x^{(n-2)}(0)-\beta x^{(n-1)}(0)=0, \\
\gamma x^{(n-2)}(1)+\delta x^{(n-1)}(1)=0,
\end{array}\right.
$$

where $\alpha, \beta, \delta, \gamma \geq 0$. He proved the existence of positive solutions under the assumption $\Delta=\beta \delta+\delta \alpha+\alpha \gamma>0$ and the following assumptions on $f$ :

2010 Mathematics Subject Classification: Primary 34B10; Secondary 34B15.

Key words and phrases: higher order $p$-Laplacian differential equation, fixed point theorem, growth condition. 
(D1) either

$$
\begin{aligned}
& \frac{\left\|f\left(t, x_{0}, \ldots, x_{n-2}\right)\right\|}{\sum_{i=0}^{n-2}\left\|x_{i}\right\|} \rightarrow 0 \quad \text { as } \sum_{i=0}^{n-2}\left\|x_{i}\right\| \rightarrow 0, \\
& \frac{\left\|f\left(t, x_{0}, \ldots, x_{n-2}\right)\right\|}{\sum_{i=0}^{n-2}\left\|x_{i}\right\|} \rightarrow+\infty \quad \text { as } \sum_{i=0}^{n-2}\left\|x_{i}\right\| \rightarrow \infty,
\end{aligned}
$$

or

$$
\begin{array}{ll}
\frac{\left\|f\left(t, x_{0}, \ldots, x_{n-2}\right)\right\|}{\sum_{i=0}^{n-2}\left\|x_{i}\right\|} \rightarrow+\infty \quad \text { as } \sum_{i=0}^{n-2}\left\|x_{i}\right\| \rightarrow 0, \\
\frac{\left\|f\left(t, x_{0}, \ldots, x_{n-2}\right)\right\|}{\sum_{i=0}^{n-2}\left\|x_{i}\right\|} \rightarrow 0 & \text { as } \sum_{i=0}^{n-2}\left\|x_{i}\right\| \rightarrow \infty .
\end{array}
$$

In [2] and [9], Agarwal and Wong investigated BVP (1). Let

$$
M=\min _{s \in[0,1], t \in[1 / 4,1 / 2]} \frac{k(t, s)}{k(s, s)},
$$

where $k(t, s)$ is the Green's function of the differential equation $-u^{\prime \prime}(t)=0$, $t \in(0,1)$, subject to the boundary conditions $\alpha u(0)-\beta u^{\prime}(0)=0$ and $\gamma u(1)+\delta u^{\prime}(1)=0$. The following existence result was established by using the upper and lower solution method.

\section{Theorem (Wong [10]). Suppose that}

(D5) there exists a function $g \in C\left([0,1] \times[0, \infty)^{n-1} ;[0, \infty)\right)$ which satisfies

$$
\begin{gathered}
f(t, 0,0, \ldots, 0) \geq 0 \quad \text { on }[0,1]\left(f \text { may be negative for } u_{i} \neq 0\right), \\
g\left(t,\left|u_{1}\right|, \ldots,\left|u_{n-1}\right|\right) \geq f\left(t, u_{1}, \ldots, u_{n-1}\right) \quad \text { on }[0,1] \times \mathbb{R}^{n-1},
\end{gathered}
$$

and one of the following:

(D6) $\max g_{0}=A_{1} \in\left[0, D_{1}\right)$ and $\min g_{\infty}=A_{2} \in\left(D_{2} / M, \infty\right]$,

(D7) $\min g_{0}=A_{3} \in\left(D_{2} / M, \infty\right]$ and $\max g_{\infty}=A_{4} \in\left[0, D_{1}\right)$,

(D8) there exist $h \in C\left([0, \infty)^{n-1} ;[0, \infty)\right)$, increasing with respect to $u_{n-1} \in[0, \infty)$, and $q \in C([0,1] ;[0, \infty))$ such that

$$
\left\{\begin{array}{l}
g\left(t, u_{1}, \ldots, u_{n-1}\right):=q(t) h\left(u_{1}, \ldots, u_{n-1}\right) \quad \text { on }[0,1] \times[0, \infty)^{n-1} \\
\sup _{u_{n-1} \in(0, \infty)} \min _{\left(u_{1}, \ldots, u_{n-2}\right) \in[0, \infty)} \frac{u_{n-1}}{Q h\left(u_{1}, \ldots, u_{n-1}\right)}>1
\end{array}\right.
$$


where

$$
\begin{gathered}
\max g_{0}:=\lim _{u_{1}, \ldots, u_{n-1} \rightarrow 0^{+}} \max _{t \in[0,1]} \frac{g\left(t, u_{1}, \ldots, u_{n-1}\right)}{u_{n-1}}, \\
\min g_{0}:=\lim _{u_{1}, \ldots, u_{n-1} \rightarrow 0^{+}} \min _{t \in[1 / 2,3 / 4]} \frac{g\left(t, u_{1}, \ldots, u_{n-1}\right)}{u_{n-1}}, \\
\max g_{\infty}:=\lim _{u_{1}, \ldots, u_{n-1} \rightarrow \infty} \max _{t \in[0,1]} \frac{g\left(t, u_{1}, \ldots, u_{n-1}\right)}{u_{n-1}}, \\
\min g_{\infty}:=\min _{u_{1}, \ldots, u_{n-1} \rightarrow \infty} \frac{g\left(t, u_{1}, \ldots, u_{n-1}\right)}{u_{n-1}}, \\
\left(\int_{t \in[1 / 2,3 / 4]}^{1} k(s, s) d s\right)^{-1}=: D_{1}=\frac{6 \rho}{6 \delta \beta+3 \gamma \beta+\alpha \gamma+3 \alpha \delta} \\
\left.\int_{1 / 2}^{3 / 4} k\left(\frac{1}{2}, s\right) d s\right)^{-1}:=D_{2}=\frac{64 \rho}{16 \beta \delta+6 \beta \gamma+3 \alpha \gamma+8 \alpha \delta}
\end{gathered}
$$

and

$$
Q:=\max _{t \in[0,1]} \int_{0}^{1} k(t, s) q(s) d s .
$$

Then BVP (1) has at least one nonnegative solution.

In [6], Lian and Wong studied the BVP for the nonlinear $p$-Laplacian differential equation of the form

$$
\left\{\begin{array}{l}
{\left[\phi\left(x^{(n-1)}(t)\right]^{\prime}+f\left(t, x(t), x^{\prime}(t), \ldots, x^{(n-2)}(t)\right)=0, \quad 0<t<1\right.} \\
x^{(i)}(0)=0 \quad \text { for } i=0,1, \ldots, n-3 \\
x^{(n-2)}(0)-B_{0}\left(x^{(n-1)}(0)\right)=0 \\
x^{(n-2)}(1)+B_{1}\left(x^{(n-1)}(1)\right)=0
\end{array}\right.
$$

We note that the boundary conditions in (2) are nonlinear, unlike the ones in (1). For BVP (2), the following existence result was established by using the fixed point theorem in cones in a suitable Banach space.

Theorem (Lian and Wong [6]). Suppose that

(D2) $\phi \in C^{1}(\mathbb{R}, \mathbb{R})$ is odd and convex and strictly increasing on $[0, \infty)$;

(D3) $f \in C\left([0,1] \times[0, \infty)^{n-1},[0, \infty)\right)$ and there exist distinct positive constants $\lambda$ and $\eta$ such that $f\left(t, u_{1}, \ldots, u_{n-1}\right) \leq \phi(\lambda /(\theta+1))$ on $[0,1] \times[0, \lambda]^{n-1}$ and $f\left(t, u_{1}, \ldots, u_{n-1}\right) \geq \phi(128 \eta)$ on $[1 / 2,3 / 4] \times$ $\left[\eta /\left(4^{n-1}(n-2) !\right), \eta\right]^{n-1}$;

(D4) $B_{0}$ and $B_{1}$ are both increasing continuous, odd functions defined on $\mathbb{R}$ and at least one of them satisfies the condition that there is $a \theta>0$ such that $0 \leq B_{i}(x) \leq \theta x$ for all $x \geq 0$. 
Then BVP (2) has at least one positive solution $x$ such that $\|x\|$ lies between $\lambda$ and $\eta$.

We note that the nonlinearity $f$ of the equation in the above mentioned papers only depends on $t, x, x^{\prime}, \ldots, x^{(n-2)}$ and the growth condition imposed on $f$ is at most linear. In Wong's theorem above, it is not easy to check the existence of $\max g_{0}, \min g_{0}, \max g_{\infty}$, and $\min g_{\infty}$; on the other hand, if one of them does not exist, BVP (1) cannot be solved.

To get solutions of a boundary value problem for the differential equation in [1], the authors proved that the right focal boundary value problem for the higher order differential equation

$$
\left\{\begin{array}{l}
(-1)^{n-p} x^{(n)}(t)=f\left(t, x(t), \ldots, x^{(n-1)}(t)\right), \quad 0<t<1, \\
x^{(i)}(0)=0 \quad \text { for } i=0,1, \ldots, p \\
x^{(i)}(1)=0 \quad \text { for } i=p+1, \ldots, n-1,
\end{array}\right.
$$

has solutions under some assumptions. The main condition imposed on $f$ is the following at most linear growth condition:

(D9) there exist nonnegative numbers $a_{i}$ and $L$ such that

$$
\left|f\left(t, x_{0}, \ldots, x_{n-1}\right)\right| \leq L+\sum_{i=0}^{n-1} a_{i}\left|x_{i}\right| .
$$

In [8], Liu studied the following BVP for a higher-order differential equation:

$$
\left\{\begin{array}{l}
x^{(n)}(t)=f\left(t, x(t), x^{\prime}(t), \ldots, x^{(n-1)}(t)\right)+r(t), \quad 0<t<1 \\
x^{(i)}(0)=0 \text { for } i=0,1, \ldots, p-2 \\
\alpha x^{(p-1)}(0)-\beta x^{(p)}(0)=\gamma x^{(p)}(1)+\tau x^{(p-1)}(1)=0 \\
x^{(i)}(1)=0 \text { for } i=p+1, \ldots, n-1,
\end{array}\right.
$$

where $1 \leq p \leq n-2, \alpha, \beta, \gamma$ and $\tau$ are constants, $f$ is continuous, and $r \in L^{1}[0,1]$. The main assumptions imposed on $f$ are as follows:

(D10) there exist continuous functions $h:[0,1] \times \mathbb{R}^{n} \rightarrow \mathbb{R}$, continuous functions $g_{i}:[0,1] \times \mathbb{R} \rightarrow \mathbb{R}(i=0,1, \ldots, n-1)$ and positive numbers $\bar{\beta}$ and $m$ such that

$$
f\left(t, x_{0}, x_{1}, \ldots, x_{n-1}\right)=h\left(t, x_{0}, x_{1}, \ldots, x_{n-1}\right)+\sum_{i=0}^{n-1} g_{i}\left(t, x_{i}\right)
$$

and

$$
x_{n-1} h\left(t, x_{0}, x_{1}, \ldots, x_{n-1}\right) \geq \bar{\beta}\left|x_{n-1}\right|^{m+1}
$$


for all $t \in[0,1]$ and $\left(x_{0}, x_{1}, \ldots, x_{n-1}\right) \in \mathbb{R}^{n}$, and

$$
\lim _{|x| \rightarrow \infty} \sup _{t \in[0,1]} \frac{\left|g_{i}(t, x)\right|}{|x|^{m}}=r_{i} \in[0, \infty) \quad \text { for } i=0,1, \ldots, n-1 ;
$$

(D11) there exist continuous functions $h\left(t, x_{0}, \ldots, x_{n-1}\right)$ and continuous functions $g_{i}:[0,1] \times \mathbb{R} \rightarrow \mathbb{R}(i=0,1, \ldots, n-1)$ such that

$$
f\left(t, x_{0}, x_{1}, \ldots, x_{n-1}\right)=h\left(t, x_{0}, \ldots, x_{n-1}\right)+\sum_{i=0}^{n-1} g_{i}\left(t, x_{i}\right)
$$

and

$$
x_{n-1} h\left(t, x_{0}, \ldots, x_{n-1}\right) \geq 0
$$

for all $t \in[0,1]$ and $\left(x_{0}, x_{1}, \ldots, x_{n-1}\right) \in \mathbb{R}^{n}$, and

$$
\lim _{|x| \rightarrow \infty} \sup _{t \in[0,1]} \frac{\left|g_{i}(t, x)\right|}{|x|}=r_{i} \in[0, \infty) \quad \text { for } i=0,1, \ldots, n-1
$$

(D12) $\alpha, \beta, \gamma, \tau \in \mathbb{R}$ with $\mu=\beta \tau+\alpha \gamma+\alpha \tau \neq 0$.

We note that the boundary conditions in the above mentioned papers are either homogeneous or satisfy (D4), and the conditions imposed on $f$ are not easy to check (see (D3), (D5) and (D8)).

Motivated by the above mentioned papers, we are concerned with the following nonlinear Sturm-Liouville boundary value problem for a higherorder differential equation with the $p$-Laplacian operator:

$$
\left\{\begin{array}{l}
{\left[\phi\left(x^{(n-1)}(t)\right)\right]^{\prime}=f\left(t, x(t), x^{\prime}(t), \ldots, x^{(n-2)}(t)\right), \quad t \in[0,1]} \\
x^{(i)}(0)=0 \text { for } i=0,1, \ldots, n-3 \\
\alpha x^{(n-2)}(0)-\beta B_{0}\left(x^{(n-1)}(0)\right)=A \\
\gamma x^{(n-2)}(1)+\tau B_{1}\left(x^{(n-1)}(1)\right)=B
\end{array}\right.
$$

where $A, B \in \mathbb{R}, \alpha, \gamma, \beta$ and $\tau$ are positive numbers, $f:[0,1] \times \mathbb{R}^{n} \rightarrow \mathbb{R}$ is continuous, $\phi(x)=|x|^{p-2} x$ with its inverse function $\psi(x)=|x|^{q-2} x$ with $1 / p+1 / q=1, B_{0}, B_{1}: \mathbb{R} \rightarrow \mathbb{R}$ are continuous. The boundary conditions in (5) are nonhomogeneous.

Our purpose is to establish the existence of solutions of BVP (5) without the assumptions (D1), (D3)-(D5), (D8) or (D9).

This paper is organized as follows. In Section 2, we present existence results for solutions of BVP (5). We also give some examples to illustrate the main results. In Section 3, the proofs of the main results are given.

2. Main results and examples. In this section, we first present sufficient conditions for the existence of solutions of BVP (5). Then examples are given to illustrate the main results. 
To present the results, we set the following assumptions, which will be used in the main results.

(A1) There exist continuous functions $h:[0,1] \times \mathbb{R}^{n-1} \rightarrow \mathbb{R}$, continuous functions $g_{i}:[0,1] \times \mathbb{R} \rightarrow \mathbb{R}(i=0,1, \ldots, n-2)$ and positive numbers $\bar{\beta}$ and $m$ such that

$$
f\left(t, x_{0}, x_{1}, \ldots, x_{n-2}\right)=h\left(t, x_{0}, x_{1}, \ldots, x_{n-2}\right)+\sum_{i=0}^{n-2} g_{i}\left(t, x_{i}\right)
$$

and

$$
x_{n-2} h\left(t, x_{0}, x_{1}, \ldots, x_{n-2}\right) \geq \bar{\beta}\left|x_{n-2}\right|^{m+1}
$$

for all $t \in[0,1]$ and $\left(x_{0}, x_{1}, \ldots, x_{n-2}\right) \in \mathbb{R}^{n-1}$, and

$$
\lim _{|x| \rightarrow \infty} \sup _{t \in[0,1]} \frac{\left|g_{i}(t, x)\right|}{|x|^{m}}=r_{i} \in[0, \infty) \quad \text { for } i=0,1, \ldots, n-2 .
$$

(A2) $\alpha>0, \beta \geq 0, \gamma>0, \tau \geq 0$ are constants.

(A3) $x B_{i}(x) \geq 0$ for all $x \in \mathbb{R}$, and there exist constants $\theta>0$ and $H>0$ such that

$$
\frac{B_{i}(x)}{\phi(x)} \geq \theta \quad \text { for }|x|>H, i=0,1 .
$$

TheOrEm 2.1. Suppose (A1)-(A3) hold. Then BVP (5) has at least one solution provided

$$
\sum_{i=0}^{n-3} \frac{r_{i}}{[(n-i-3) !]^{m}}+r_{n-2}<\bar{\beta} .
$$

REMARK 2.2. Condition (A1) is imposed on the nonlinearity $f$, and (A3) is imposed on $B_{0}$ and $B_{1}$. They are different from known ones since the growth is allowed to be superlinear (the degrees of phase variables are allowed to be greater than 1 if $f, B_{0}, B_{1}$ are polynomials).

REMARK 2.3. Theorem 2.1 is new since

(i) we allow $f$ to depend on $t, x, x^{\prime}, \ldots, x^{(n-2)}$ and the degree of the variables in $f$ can be greater than 1 if $f$ is a polynomial;

(ii) the conditions imposed on $B_{0}$ and $B_{1}$ are weaker than the known ones (see (D4)), since we allow $B_{0}$ and $B_{1}$ to be superlinear, and the monotonicity property of $B_{0}$ and $B_{1}$ is not needed;

(iii) the methods of proof are different from the known ones, since the considerably technical assumptions (D1), (D3), (D4), (D5), (D8) and (D9) are not used;

(iv) the assumptions here are easy to check.

The proof of Theorem 2.1 is given in Section 3. Now, we present some examples to illustrate the main result. In [1], BVP (3) is studied under the 
assumption (D9) in which the growth condition imposed on $f$ is at most linear. This motivates us to study BVPs with superlinear nonlinearities.

EXAmple 2.4. Consider the problem

$$
\left\{\begin{aligned}
{\left[\phi\left(x^{(3)}(t)\right)\right]=} & \left(2+x^{2}(t)+2\left[x^{\prime}(t)\right]^{2}+\left[x^{\prime \prime}(t)\right]^{4}\right)\left[x^{\prime \prime}(t)\right]^{3} \\
& +\sum_{i=0}^{2} a_{i}\left[x^{(i)}(t)\right]^{3}+r(t), \\
x(0)=x^{\prime}(0)= & 0, \quad x^{\prime \prime}(0)-2\left[x^{\prime \prime \prime}(0)\right]^{3}=x^{\prime \prime}(1)+8\left[x^{\prime \prime \prime}(1)\right]^{5}=2,
\end{aligned}\right.
$$

where $r(t)$ is a continuous function, $\phi(x)=|x|^{p-2} x$ with $p>1$. It corresponds to BVP (5) with $n=4, f\left(t, x_{0}, x_{1}, x_{2}\right)=\left(2+x_{0}^{2}+2 x_{1}^{2}+x_{2}^{4}\right) x_{2}^{3}+\sum_{i=0}^{2} a_{i} x_{i}^{3}+$ $r(t), \alpha=\gamma=1, \beta=2, \tau=8, B_{0}(x)=x^{3}$ and $B_{1}(x)=x^{5}, A=B=2$.

Choose $m=3, h\left(t, x_{0}, x_{1}, x_{2}\right)=\left(2+x_{0}^{2}+2 x_{1}^{2}+x_{2}^{4}\right) x_{2}^{3}, g_{i}\left(t, x_{i}\right)=$ $a_{i} x_{i}^{3}+r(t) / 3, i=0,1,2$. One sees that

$$
\begin{gathered}
f\left(t, x_{0}, x_{1}, x_{2}\right)=h\left(t, x_{0}, x_{1}, x_{2}\right)+g_{0}\left(t, x_{0}\right)+g_{1}\left(t, x_{1}\right)+g_{2}\left(t, x_{2}\right), \\
h\left(t, x_{0}, x_{1}, x_{2}\right) x_{2}=\left(2+x_{0}^{2}+2 x_{1}^{2}+x_{2}^{4}\right) x_{2}^{4} \geq 2 x_{2}^{4},
\end{gathered}
$$

and

$$
\lim _{x_{i} \rightarrow \infty} \frac{\left|g_{i}\left(t, x_{i}\right)\right|}{x_{i}^{m}}=\left|a_{i}\right|, \quad i=0,1,2 .
$$

Hence (A1) holds. It is easy to see that (A3) holds. Since

$$
\frac{B_{0}(x)}{\phi(x)}=\frac{x^{3}}{|x|^{p-2} x}=|x|^{4-p}, \quad \frac{B_{1}(x)}{\phi(x)}=\frac{x^{5}}{|x|^{p-2} x}=|x|^{6-p},
$$

one sees that (A4) holds, i.e., there exist constants $\theta=1$ and $H>0$ such that $B_{i}(x) / \phi(x) \geq \theta$ for all $|x|>H(i=0,1)$. By Theorem 2.1, it is easy to check that, for each $r \in C^{0}[0,1]$, BVP (7) has at least one solution if $\sum_{i=0}^{2}\left|a_{i}\right|<2$.

EXAMPle 2.5. Consider the problem

$$
\left\{\begin{aligned}
& {\left[\phi\left(x^{(5)}(t)\right)\right]^{\prime}=}\left(2+x^{2}(t)+2\left[x^{\prime}(t)\right]^{2}+\left[x^{\prime \prime \prime}(t)\right]^{4}\right)\left[x^{(4)}(t)\right]^{5} \\
&+\sum_{i=0}^{4} a_{i}\left[x^{(i)}(t)\right]^{5}+r(t) \\
& x(0)=x^{\prime}(0)= x^{\prime \prime}(0)=x^{\prime \prime \prime}(0)=0 \\
& x^{\prime \prime \prime \prime}(0)-2\left[x^{\prime \prime \prime \prime \prime}(0)\right]^{5}=x^{\prime \prime \prime \prime}(1)+8\left[x^{\prime \prime \prime \prime \prime}(1)\right]^{7}=8 \\
& x^{(5)}(1)=0 .
\end{aligned}\right.
$$

By Theorem 2.1, it is easy to check that, for each $r \in C^{0}[0,1]$, BVP (8) has at least one solution if

$$
\frac{\left|a_{0}\right|}{6^{5}}+\frac{\left|a_{1}\right|}{2^{5}}+\left|a_{2}\right|+\left|a_{3}\right|+\left|a_{4}\right|<2 .
$$


EXAMPLE 2.6. Consider the problem

$$
\left\{\begin{array}{l}
{\left[\left(x^{(4)}(t)\right)^{3 / 2}\right]^{\prime}=\frac{2+\left[x^{\prime}(t)\right]^{2}}{1+\left[x^{\prime}(t)\right]^{2}}\left[x^{\prime \prime \prime}(t)\right]^{3}+a[x(t)]^{3}+b\left[x^{\prime}(t)\right]^{3}+r(t),} \\
x(0)=x^{\prime}(0)=x^{\prime \prime}(0)=0 \\
x^{\prime \prime \prime}(0)-2\left[x^{\prime \prime \prime \prime}(0)\right]^{3}=x^{\prime \prime \prime}(1)+3 x^{\prime \prime \prime \prime}(1)=9 .
\end{array}\right.
$$

By Theorem 2.1, it is easy to check that, for each $r \in C^{0}[0,1]$, BVP (9) has at least one solution if $|a|+|b|<1$.

It is of interest that all nonlinear functions in Examples 2.1-2.3 are superlinear, so the known results in [1, 2, 17, 8, 9] mentioned in Section 1 cannot be applied.

3. The proof of the main theorem. In this section, we prove the theorem presented in Section 2. This will be done by using the following fixed point theorem.

Let $X$ and $Y$ be real Banach spaces, $L: D(L) \subset X \rightarrow Y$ be a Fredholm operator of index zero, and $P: X \rightarrow X, Q: Y \rightarrow Y$ be projectors such that $\operatorname{Im} P=\operatorname{Ker} L, \quad \operatorname{Ker} Q=\operatorname{Im} L, \quad X=\operatorname{Ker} L \oplus \operatorname{Ker} P, \quad Y=\operatorname{Im} L \oplus \operatorname{Im} Q$. It follows that

$$
\left.L\right|_{D(L) \cap \operatorname{Ker} P}: D(L) \cap \operatorname{Ker} P \rightarrow \operatorname{Im} L
$$

is invertible. We denote its generalized inverse by $K_{P}: \operatorname{Im} L \rightarrow D(L) \cap \operatorname{Ker} P$.

If $\Omega$ is an open bounded subset of $X$ with $D(L) \cap \bar{\Omega} \neq \emptyset$, a map $N: X \rightarrow$ $Y$ will be called $L$-compact on $\bar{\Omega}$ if $Q N(\bar{\Omega})$ is bounded and $K_{P}(I-Q) N$ : $\bar{\Omega} \rightarrow X$ is compact.

Lemma 3.1. Let $X$ and $Y$ be real Banach spaces, $L: X \rightarrow Y$ be a Fredholm operator of index zero and let $N: X \rightarrow Y$ be L-compact on each open bounded $\Omega \subset X$. Assume that the following conditions are satisfied:

(i) $L x \neq \lambda N x$ for every $(x, \lambda) \in[(D(L) \backslash \operatorname{Ker} L) \cap \partial \Omega] \times(0,1)$;

(ii) $N x \notin \operatorname{Im} L$ for every $x \in \operatorname{Ker} L \cap \partial \Omega$;

(iii) $\operatorname{deg}\left(\left.\Lambda Q N\right|_{\operatorname{Ker} L}, \Omega \cap \operatorname{Ker} L, 0\right) \neq 0$ for some isomorphism $\Lambda: Y / \operatorname{Im} L$ $\rightarrow \operatorname{Ker} L$.

Then the equation $L x=N x$ has at least one solution in $D(L) \cap \bar{\Omega}$.

It is easy to transform BVP (5) to the system

$$
\begin{aligned}
& \left\{\begin{array}{l}
x^{(n-1)}(t)=\psi(y(t)), \\
y^{\prime}(t)=f\left(t, x(t), x^{\prime}(t), \ldots, x^{(n-2)}(t)\right), \quad t \in[0,1],
\end{array}\right. \\
& \left\{\begin{array}{l}
x^{(i)}(0)=0 \quad \text { for } i=0,1, \ldots, n-3, \\
\alpha x^{(n-2)}(0)-\beta B_{0}(\psi(y(0)))=A, \\
\gamma x^{(n-2)}(1)+\tau B_{1}(\psi(y(1)))=B .
\end{array}\right.
\end{aligned}
$$


For $x \in C^{0}[0,1]$, define $\|x\|_{\infty}=\max _{t \in[0,1]}|x(t)|$. Let $X=C^{n-2}[0,1] \times$ $C^{0}[0,1]$ and $Y=C^{0}[0,1] \times C^{0}[0,1] \times \mathbb{R}^{2}$. We endow $X$ with the norm

$$
\|x\|=\max \left\{\left\|x_{1}\right\|_{\infty}, \ldots,\left\|x_{1}^{(n-2)}\right\|_{\infty},\left\|x_{2}\right\|_{\infty}\right\}
$$

for all $x=\left(x_{1}, x_{2}\right) \in X$, and $Y$ with the norm

$$
\|y\|=\max \left\{\left\|y_{1}\right\|_{\infty},\left\|y_{2}\right\|_{\infty},\left|a_{1}\right|,\left|a_{2}\right|\right\}
$$

for $y=\left(y_{1}, y_{2}, a_{1}, a_{2}\right) \in Y$. Then $X$ and $Y$ are real Banach spaces.

For BVP (10)-(11), let

$$
\begin{aligned}
D(L)=\left\{(x, y) \in X: x \in C^{n-1}(0,1), y\right. & \in C^{1}(0,1), \\
& \left.x^{(i)}(0)=0, i=0, \ldots, n-3\right\} .
\end{aligned}
$$

Define the linear operator $L: X \cap D(L) \rightarrow Y$ and the nonlinear operator $N: X \rightarrow Y$ by

$$
\begin{aligned}
L\left(\begin{array}{l}
x(t) \\
y(t)
\end{array}\right) & =\left(\begin{array}{c}
c x^{(n-1)}(t) \\
y^{\prime}(t) \\
x^{(n-2)}(0) \\
x^{(n-2)}(1)
\end{array}\right) \quad \text { for }(x, y) \in X \cap D(L), \\
N\left(\begin{array}{l}
x(t) \\
y(t)
\end{array}\right) & =\left(\begin{array}{c}
c \psi(y(t)) \\
f\left(t, x(t), x^{\prime}(t), \ldots, x^{(n-2)}\right) \\
(\beta / \alpha) B_{0}(\psi(y(0)))+A / \alpha \\
-(\tau / \gamma) B_{1}(\psi(y(1)))+B / \gamma
\end{array}\right) \quad \text { for }(x, y) \in X .
\end{aligned}
$$

Lemma 3.2. Suppose that (A3) holds. Then

(i) $\operatorname{Ker} L=\{(0, a): a \in \mathbb{R}\}$ and $\operatorname{Im} L=\left\{\left(y_{1}, y_{2}, a, b\right): \int_{0}^{1} y_{1}(s) d s=\right.$ $b-a\}$

(ii) $x$ is a solution of $B V P(5)$ if $(x, y) \in D(L)$ is a solution of the operator equation $L(x, y)=N(x, y)$ in $D(L)$;

(iii) there exist projectors $P: X \rightarrow X$ and $Q: Y \rightarrow Y$ such that $\operatorname{Ker} L=$ $\operatorname{Im} P$ and $\operatorname{Ker} Q=\operatorname{Im} L$; furthermore, if $\Omega \subset X$ is an open bounded subset with $\bar{\Omega} \cap D(L) \neq \emptyset$, then $N$ is $L$-compact on $\bar{\Omega}$;

(iv) $L$ is a Fredholm operator of index zero.

Proof. The proofs of (i) and (ii) are simple.

For (iii), we define the projectors $P$ and $Q$ by

$$
P(x, y)=(0, y(0)) \quad \text { for all } x=(x, y) \in X,
$$

$Q\left(y_{1}, y_{2}, a, b\right)=\left(\int_{0}^{1} y_{1}(s) d s-(b-a), 0,0,0\right) \quad$ for all $\left(y_{1}, y_{2}, a, b\right) \in Y$. 
The generalized inverse $K_{P}: \operatorname{Im} L \rightarrow D(L) \cap \operatorname{Ker} P$ of $L$ is

$$
K_{P}\left(y_{1}, y_{2}, a, b\right)=\left(\int_{0}^{t} \frac{(t-s)^{n-2}}{(n-2) !} y_{1}(s) d s+\frac{a t^{n-2}}{(n-2) !}, \int_{0}^{t} y_{2}(s) d s\right),
$$

and the isomorphism $\Lambda: Y / \operatorname{Im} L \rightarrow \operatorname{Ker} L$ is defined by $\Lambda(a, 0,0,0)=(0, a)$.

The proof of (iv) is standard, using the methods of [10].

Proof of Theorem 2.1. From the definitions of $X, Y, D(L)$ and the operators $L$ and $N$, it is easy to show that $L: D(L) \subset X \rightarrow Y$ is a Fredholm operator of index zero, and $N: X \rightarrow Y$ is $L$-compact on any open bounded subset of $X$. To apply Lemma 3.1, we proceed in the following four steps.

STEP 1. Let

$$
\Omega_{0}=\{(x, y) \in D(L): L(x, y)=\lambda N(x, y) \text { for some } \lambda \in(0,1)\} .
$$

We prove that $\Omega_{0}$ is bounded. It suffices to prove that there exists a constant $M_{5}>0$ such that

$$
\begin{aligned}
\|(x, y)\|=\max \left\{\|x\|_{\infty}, \ldots,\left\|x^{(n-2)}\right\|_{\infty},\|y\|_{\infty}\right\} & \leq M_{5} \\
& \text { for all }(x, y) \in \Omega_{0} .
\end{aligned}
$$

(i) We prove that there exists a constant $M>0$ such that

$$
\int_{0}^{1}\left|x^{(n-2)}(s)\right|^{m+1} d s \leq M .
$$

For $(x, y) \in \Omega_{0}$, we have

$$
\left\{\begin{array}{l}
x^{(n-1)}(t)=\lambda \psi(y(t)) \\
y^{\prime}(t)=\lambda f\left(t, x(t), x^{\prime}(t), \ldots, x^{(n-2)}(t)\right), \quad t \in[0,1] \\
x^{(i)}(0)=0 \text { for } i=0,1, \ldots, n-3, \\
x^{(n-2)}(0)=\lambda(\beta / \alpha) B_{0}(\psi(y(0)))+\lambda A / \alpha \\
x^{(n-2)}(1)=-\lambda(\tau / \gamma) B_{1}(\psi(y(1)))+\lambda B / \gamma .
\end{array}\right.
$$

It follows from (14) that

$$
\left[\phi\left(\frac{x^{(n-1)}(t)}{\lambda}\right)\right]^{\prime}=\lambda f\left(t, x(t), x^{\prime}(t), \ldots, x^{(n-2)}(t)\right) .
$$

So

$$
\begin{array}{r}
{\left[\phi\left(x^{(n-1)}(t)\right)\right]^{\prime} x^{(n-2)}(t)} \\
=\phi(\lambda) \lambda f\left(t, x(t), x^{\prime}(t), \ldots, x^{(n-2)}(t)\right) x^{(n-2)}(t) .
\end{array}
$$

Integrating (15) from 0 to 1 , we get 


$$
\begin{aligned}
& \phi(\lambda) \lambda \int_{0}^{1} f\left(s, x(s), \ldots, x^{(n-2)}(s)\right) x^{(n-2)}(s) d s \\
&=\phi\left(x^{(n-1)}(1)\right) x^{(n-2)}(1)-\phi\left(x^{(n-1)}(0)\right) x^{(n-2)}(0) \\
& \quad-\int_{0}^{1} \phi\left(x^{(n-1)}(s)\right) x^{(n-1)}(s) d s \\
&=\phi\left(x^{(n-1)}(1)\right) \lambda\left(-\frac{\tau}{\gamma} B_{1}\left(\frac{x^{(n-1)}(1)}{\lambda}\right)+\frac{B}{\gamma}\right) \\
& \quad-\phi\left(x^{(n-1)}(0)\right) \lambda\left(+\frac{\beta}{\alpha} B_{0}\left(\frac{x^{(n-1)}(0)}{\lambda}\right)+\frac{A}{\alpha}\right) \\
& \quad-\int_{0}^{1} \phi\left(x^{(n-1)}(s)\right) x^{(n-1)}(s) d s \\
&=\left(-\phi\left(x^{(n-1)}(1)\right) \frac{\tau}{\gamma} B_{1}\left(\frac{x^{(n-1)}(1)}{\lambda}\right)+\frac{B}{\gamma} \phi\left(x^{(n-1)}(1)\right)\right) \\
&-\lambda\left(\phi\left(x^{(n-1)}(0)\right) \frac{\beta}{\alpha} B_{0}\left(\frac{x^{(n-1)}(0)}{\lambda}\right)+\frac{A}{\alpha} \phi\left(x^{(n-1)}(0)\right)\right) \\
& \quad-\int_{0}^{1} \phi\left(x^{(n-1)}(s)\right) x^{(n-1)}(s) d s .
\end{aligned}
$$

It follows from (A3) and the definition of $\phi$ that

$$
\begin{aligned}
\frac{B_{i}(x / \lambda)}{\phi(x / \lambda)} & \geq \theta \quad \text { if }|x / \lambda|>H, i=0,1, \\
-\phi(x) \frac{\tau}{\gamma} B_{1}\left(\frac{x}{\lambda}\right)+\frac{B}{\gamma} \phi(x) & =-\frac{\tau}{\gamma} \phi(\lambda) \phi\left(\frac{x}{\lambda}\right) B_{1}\left(\frac{x}{\lambda}\right)+\frac{B}{\gamma} \phi(x) \\
& \leq \frac{|B|}{\gamma} \phi(\lambda) \phi(H) \quad \text { if }|x / \lambda| \leq H,
\end{aligned}
$$

and

$$
\begin{aligned}
-\phi(x) \frac{\beta}{\alpha} B_{0}\left(\frac{x}{\lambda}\right)-\frac{A}{\alpha} \phi(x) & =-\frac{\beta}{\alpha} \phi(\lambda) \phi\left(\frac{x}{\lambda}\right) B_{0}\left(\frac{x}{\lambda}\right)-\frac{A}{\alpha} \phi(x) \\
& \leq \frac{|A|}{\alpha} \phi(\lambda) \phi(H) \quad \text { if }|x / \lambda| \leq H .
\end{aligned}
$$

Then

$$
\begin{aligned}
& \lambda\left(-\phi\left(x^{(n-1)}(1)\right) \frac{\tau}{\gamma} B_{1}\left(\frac{x^{(n-1)}(1)}{\lambda}\right)+\frac{B}{\gamma} \phi\left(x^{(n-1)}(1)\right)\right) \\
& \leq\left\{\begin{array}{l}
\lambda(|B| / \gamma) \phi(\lambda) \phi(H) \text { if }\left|x^{(n-1)}(1) / \lambda\right| \leq H, \\
\lambda\left(-\phi\left(x^{(n-1)}(1)\right) \frac{\tau}{\gamma} \theta \phi\left(\frac{x^{(n-1)}(1)}{\lambda}\right)+\frac{B}{\gamma} \phi\left(x^{(n-1)}(1)\right)\right) \text { if }\left|x^{(n-1)}(1) / \lambda\right|>H,
\end{array}\right.
\end{aligned}
$$




$$
\begin{aligned}
& =\left\{\begin{array}{l}
\lambda|B| \phi(H) \quad \text { if }\left|x^{(n-1)}(1) / \lambda\right| \leq H, \\
\lambda \frac{\tau \theta}{\phi(\lambda) \gamma}\left[-\left(\phi\left(x^{(n-1)}(1)\right)-\frac{B}{\gamma} \frac{\phi(\lambda) \gamma}{2 \tau \theta}\right)^{2}+\frac{\phi(\lambda)^{2} B^{2}}{4 \tau^{2} \theta^{2}}\right] \quad \text { if }\left|x^{(n-1)}(1) / \lambda\right|>H
\end{array}\right. \\
& \leq \max \left\{\lambda \phi(\lambda)|B| \phi(H), \lambda \phi(\lambda) \frac{B^{2}}{4 \gamma \tau \theta}\right\}=: \lambda \phi(\lambda) \overline{M_{1}} .
\end{aligned}
$$

Similarly we get

$$
\begin{aligned}
\lambda\left(-\phi\left(x^{(n-1)}(0)\right)\right. & \left.\frac{\tau}{\gamma} B_{0}\left(\frac{x^{(n-1)}(0)}{\lambda}\right)+A \phi\left(x^{(n-1)}(0)\right)\right) \\
& \leq \max \left\{\lambda \phi(\lambda)|A| \phi(H), \lambda \phi(\lambda) \frac{A^{2}}{4 \beta \alpha \theta}\right\}=: \lambda \phi(\lambda) \overline{M_{0}} .
\end{aligned}
$$

Then

$$
\phi(\lambda) \lambda \int_{0}^{1} f\left(s, x(s), \ldots, x^{(n-2)}(s)\right) x^{(n-2)}(s) d s \leq \lambda \phi(\lambda) \overline{M_{1}}+\lambda \phi(\lambda) \overline{M_{0}} .
$$

It follows that

$$
\int_{0}^{1} f\left(s, x(s), \ldots, x^{(n-2)}(s)\right) x^{(n-2)}(s) d s \leq \overline{M_{1}}+\overline{M_{0}} .
$$

Using (A1) and (A2), we get

$$
\begin{aligned}
& \int_{0}^{1} f\left(s, x(s), \ldots, x^{(n-2)}(s)\right) x^{(n-2)}(s) d s \\
& \quad=\int_{0}^{1} h\left(s, x(s), \ldots, x^{(n-2)}(s)\right) x^{(n-2)}(s) d s+\sum_{i=0}^{n-2} \int_{0}^{1} g_{i}\left(s, x^{(i)}(s)\right) x^{(n-2)}(s) d s \\
& \quad \leq \overline{M_{0}}+\overline{M_{1}} .
\end{aligned}
$$

Hence

$$
\begin{aligned}
\bar{\beta} \int_{0}^{1}\left|x^{(n-2)}(s)\right|^{m+1} d s & \leq \int_{0}^{1} h\left(s, x(s), \ldots, x^{(n-2)}(s)\right) x^{(n-2)}(s) d s \\
& \leq \overline{M_{0}}+\overline{M_{1}}-\sum_{i=0}^{n-2} \int_{0}^{1} g_{i}\left(s, x^{(i)}(s)\right) x^{(n-2)}(s) d s \\
& \leq \overline{M_{0}}+\overline{M_{1}}+\sum_{i=0}^{n-2} \int_{0}^{1}\left|g_{i}\left(s, x^{(i)}(s)\right)\right|\left|x^{(n-2)}(s)\right| d s .
\end{aligned}
$$

From (6), pick $\epsilon>0$ such that

$$
\bar{\beta}>\sum_{i=0}^{n-3} \frac{r_{i}+\epsilon}{[(n-i-3) !]^{m}}+r_{n-2}+\epsilon .
$$


For such $\epsilon>0$, from (A1), there exists $\delta>0$ such that

$$
\left|g_{i}(t, x)\right| \leq\left(r_{i}+\epsilon\right)|x|^{m} \quad \text { for }|x|>\delta \text { and } t \in[0,1], i=0, \ldots, n-2 .
$$

Denote

$$
\begin{aligned}
\Delta_{1, i} & =\left\{t: t \in[0,1],\left|x^{(i)}(t)\right| \leq \delta\right\}, & & i=0, \ldots, n-2, \\
\Delta_{2, i} & =\left\{t: t \in[0,1],\left|x^{(i)}(t)\right|>\delta\right\}, & & i=0, \ldots, n-2, \\
g_{\delta, i} & =\max _{t \in[0,1],|x| \leq \delta}\left|g_{i}(t, x)\right|, & & i=0, \ldots, n-2 .
\end{aligned}
$$

We note, for $i=0, \ldots, n-3$, that

$$
\left|x^{(i)}(t)\right|=\left|\int_{0}^{t} \frac{(t-s)^{n-3-i}}{(n-3-i) !} x^{(n-2)}(s) d s\right| \leq \frac{1}{(n-3-i) !} \int_{0}^{1}\left|x^{(n-2)}(s)\right| d s .
$$

Then we get

$$
\begin{aligned}
& \bar{\beta} \int_{0}^{1}\left|x^{(n-2)}(s)\right|^{m+1} d s \leq \overline{M_{0}}+\overline{M_{1}} \\
& \quad+\sum_{i=0}^{n-2} \int_{\Delta_{1, i}}\left|g_{i}\left(s, x^{(i)}(s)\right)\right|\left|x^{(n-2)}(s)\right| d s+\sum_{i=0}^{n-2} \int_{\Delta_{2, i}}\left|g_{i}\left(s, x^{(i)}(s)\right)\right|\left|x^{(n-2)}(s)\right| d s \\
& \leq \sum_{i=0}^{n-2} g_{\delta, i}\left(\int_{0}^{1}\left|x^{(n-2)}(s)\right|^{m+1} d s\right)^{1 /(m+1)}+\left(r_{n-2}+\epsilon\right) \int_{0}^{1}\left|x^{(n-2)}(s)\right|^{m+1} d s \\
& \quad+\sum_{i=0}^{n-3} \frac{r_{i}+\epsilon}{[(n-3-i) !]^{m}} \int_{0}^{1}\left|x^{(n-2)}(s)\right|^{m+1} d s+\overline{M_{0}}+\overline{M_{1}} .
\end{aligned}
$$

One has

$$
\begin{aligned}
& \left(\bar{\beta}-\sum_{i=0}^{n-3} \frac{r_{i}+\epsilon}{[(n-3-i) !]^{m}}-\left(r_{n-2}+\epsilon\right)\right) \int_{0}^{1}\left|x^{(n-2)}(s)\right|^{m+1} d s \\
& \leq \sum_{i=0}^{n-2} g_{\delta, i}\left(\int_{0}^{1}\left|x^{(n-2)}(s)\right|^{m+1} d s\right)^{1 /(m+1)}+\overline{M_{0}}+\overline{M_{1}} .
\end{aligned}
$$

It is easy to see from the definition of $\epsilon$ that there is $M>0$ such that

$$
\int_{0}^{1}\left|x^{(n-2)}(s)\right|^{m+1} d s \leq M .
$$

(ii) We prove that there exists a constant $M_{2}>0$ such that

$$
\max _{i=0,1, \ldots, n-2}\left\|x^{(i)}\right\|_{\infty} \leq M_{2} \text {. }
$$


It follows from (i) that for $i=0, \ldots, n-3$, we get

$$
\left\|x^{(i)}\right\|_{\infty} \leq \frac{1}{(n-3-i) !} \int_{0}^{1}\left|x^{(n-2)}(s)\right| d s \leq \frac{1}{(n-3-i) !} M^{1 /(m+1)} .
$$

Now, we consider $\left\|x^{(n-2)}\right\|_{\infty}$. It follows from the above inequality that there is $t_{0} \in[0,1]$ such that $\left|x^{(n-2)}\left(t_{0}\right)\right| \leq M^{1 /(m+1)}$.

For $t \leq t_{0}$, by integrating (15) from 0 to $t$, we get

$$
\begin{aligned}
& \phi(\lambda) \lambda \int_{0}^{t} f\left(s, x(s), \ldots, x^{(n-2)}(s)\right) x^{(n-2)}(s) d s \\
& =\phi\left(x^{(n-1)}(t)\right) x^{(n-2)}(t)-\phi\left(x^{(n-1)}(0)\right) x^{(n-2)}(0)-\int_{0}^{t} \phi\left(x^{(n-1)}(s)\right) x^{(n-1)}(s) d s \\
& \leq \phi\left(x^{(n-1)}(t)\right) x^{(n-2)}(t)+\phi(\lambda) \lambda \overline{M_{0}} .
\end{aligned}
$$

Thus we have

$$
\begin{aligned}
& x^{(n-1)}(t) \psi\left(x^{(n-2)}(t)\right) \\
& \quad \geq \psi\left(\phi(\lambda) \lambda \int_{0}^{t} f\left(s, x(s), \ldots, x^{(n-2)}(s)\right) x^{(n-2)}(s) d s-\phi(\lambda) \lambda \overline{M_{0}}\right) .
\end{aligned}
$$

It follows that

$$
\begin{aligned}
& \int_{t}^{t_{0}} x^{(n-1)}(s) \psi\left(x^{(n-2)}(s)\right) d s \\
& \geq \int_{t}^{t_{0}} \psi\left(\phi(\lambda) \lambda \int_{0}^{s} f\left(u, x(u), \ldots, x^{(n-2)}(u)\right) x^{(n-2)}(u) d u-\phi(\lambda) \lambda \overline{M_{0}}\right) d s .
\end{aligned}
$$

Hence

$$
\begin{aligned}
& \frac{1}{q}\left|x^{(n-2)}(t)\right|^{q} \leq \frac{1}{q}\left|x^{(n-2)}\left(t_{0}\right)\right|^{q} \\
& \quad-\int_{t}^{t_{0}} \psi\left(\phi(\lambda) \lambda \int_{0}^{s} f\left(u, x(u), \ldots, x^{(n-2)}(u)\right) x^{(n-2)}(u) d u-\phi(\lambda) \lambda \overline{M_{0}}\right) d s \\
& \leq \frac{1}{q} M^{q /(m+1)} \\
& \quad-\phi(\lambda) \lambda \int_{t}^{t_{0}} \psi\left(\int_{0}^{s}\left(h\left(u, x(u), \ldots, x^{(n-2)}(u)\right)+\sum_{i=0}^{n-2} g_{i}\left(u, x^{(i)}(u)\right)\right)\right. \\
& \left.\times x^{(n-2)}(u) d u-\overline{M_{0}}\right) d s
\end{aligned}
$$




$$
\begin{aligned}
& \leq \frac{1}{q} M^{q /(m+1)}-\phi(\lambda) \lambda \int_{t}^{t_{0}} \psi\left(\left(\int_{0}^{s} \bar{\beta}\left|x^{(n-2)}(u)\right|^{m+1} d u+\sum_{i=0}^{n-2} \int_{0}^{s} g_{i}\left(u, x^{(i)}(u)\right)\right.\right. \\
& \left.\left.\times x^{(n-2)}(u) d u\right)-\overline{M_{0}}\right) d s \\
& \leq \frac{1}{q} M^{q /(m+1)}-\phi(\lambda) \lambda \int_{t}^{t_{0}} \psi\left(\int_{0}^{s}\left(\sum_{i=0}^{n-2} g_{i}\left(u, x^{(i)}(u)\right)\right) x^{(n-2)}(u) d u-\overline{M_{0}}\right) d s \\
& \leq \overline{M_{0}}+\frac{1}{q} M^{q /(m+1)}+\psi\left(\int_{0}^{1} \sum_{i=0}^{n-2}\left|g_{i}\left(u, x^{(i)}(u)\right)\right|\left|x^{(n-2)}(u)\right| d u\right) \\
& \leq \overline{M_{0}}+\frac{1}{q} M^{q /(m+1)}+\psi\left(\sum_{i=0}^{n-2} g_{\delta, i} \int_{0}^{1}\left|x^{(n-2)}(s)\right| d s\right. \\
& \left.+\sum_{i=0}^{n-3} \frac{r_{i}+\epsilon}{[(n-3-i) !]^{m}}\left(\int_{0}^{1}\left|x^{(n-2)}(u)\right| d u\right)^{m+1}+\left(r_{n-2}+\epsilon\right) \int_{0}^{1}\left|x^{(n-2)}(s)\right|^{m+1} d s\right) \\
& \leq \overline{M_{0}}+\frac{1}{q} M^{q /(m+1)}+\psi\left(\sum_{i=0}^{n-2} g_{\delta, i}\left(\int_{0}^{1}\left|x^{(n-2)}(s)\right|^{m+1} d s\right)^{1 /(m+1)}\right. \\
& \left.+\sum_{i=0}^{n-3} \frac{r_{i}+\epsilon}{[(n-3-i) !]^{m}} \int_{0}^{1}\left|x^{(n-2)}(u)\right|^{m+1} d u+\left(r_{n-2}+\epsilon\right) \int_{0}^{1}\left|x^{(n-2)}(s)\right|^{m+1} d s\right) \\
& \leq \overline{M_{0}}+\frac{1}{q} M^{q /(m+1)} \\
& +\psi\left(\sum_{i=0}^{n-2} g_{\delta, i} M^{1 /(m+1)}+\sum_{i=0}^{n-3} \frac{r_{i}+\epsilon}{[(n-3-i) !]^{m}} M+\left(r_{n-2}+\epsilon\right) M\right) .
\end{aligned}
$$

It follows that

$$
\begin{aligned}
\frac{\left|x^{(n-2)}(t)\right|^{q}}{q} & \leq \overline{M_{0}}+\frac{M^{q /(m+1)}}{q} \\
& +\psi\left(\sum_{i=0}^{n-2} g_{\delta, i} M^{1 /(m+1)}+\sum_{i=0}^{n-3} \frac{M\left(r_{i}+\epsilon\right)}{[(n-3-i) !]^{m}}+\left(r_{n-2}+\epsilon\right) M\right) .
\end{aligned}
$$

For $t \geq t_{0}$, we have

$$
\begin{aligned}
& \phi(\lambda) \lambda \int_{t}^{1} f\left(s, x(s), \ldots, x^{(n-2)}(s)\right) x^{(n-2)}(s) d s \\
& =\phi\left(x^{(n-1)}(1)\right) x^{(n-2)}(1)-\phi\left(x^{(n-1)}(t)\right) x^{(n-2)}(t)-\int_{t}^{1} \phi\left(x^{(n-1)}(s)\right) x^{(n-1)}(s) d s \\
& \leq-\phi\left(x^{(n-1)}(t)\right) x^{(n-2)}(t)+\phi(\lambda) \lambda \overline{M_{1}} .
\end{aligned}
$$


Thus we have

$$
\begin{aligned}
& x^{(n-1)}(t) \psi\left(x^{(n-2)}(t)\right) \\
& \quad \leq-\psi\left(\phi(\lambda) \lambda \int_{t}^{1} f\left(u, x(u), \ldots, x^{(n-2)}(u)\right) x^{(n-2)}(u) d u+\phi(\lambda) \lambda \overline{M_{1}}\right) .
\end{aligned}
$$

We get

$$
\begin{aligned}
& \frac{1}{q}\left|x^{(n-2)}(t)\right|^{q} \leq \frac{1}{q}\left|x^{(n-2)}\left(t_{0}\right)\right|^{q} \\
& \quad-\int_{t_{0}}^{t} \psi\left(\phi(\lambda) \lambda \int_{s}^{1} f\left(u, x(u), \ldots, x^{(n-2)}(u)\right) x^{(n-2)}(u) d u+\phi(\lambda) \lambda \overline{M_{1}}\right) d s .
\end{aligned}
$$

Similarly to the above argument, we can get

$$
\begin{aligned}
\frac{\left|x^{(n-2)}(t)\right|^{q}}{q} \leq & \overline{M_{1}}+\frac{M^{1 /(m+1)}}{q}+\sum_{i=0}^{n-2} g_{\delta, i} M^{1 /(m+1)} \\
& +\sum_{i=0}^{n-3} \frac{M\left(r_{i}+\epsilon\right)}{[(n-3-i) !]^{m}}+\left(r_{n-2}+\epsilon\right) M .
\end{aligned}
$$

It follows from (18) and (19) that there exists a constant $M_{1}>0$ such that $\left\|x^{(n-2)}\right\|_{\infty} \leq M_{1}$. Together with (17), one gets

$$
\|x\|=\max _{i=0, \ldots, n-2}\left\|x^{(i)}\right\|_{\infty} \leq \max \left\{M_{1}, \frac{M^{1 /(m+1)}}{(n-3-i) !}\right\}=: M_{2} .
$$

(iii) We prove that there exist constants $M_{3}, M_{4}>0$ such that

$$
\|y\|_{\infty} \leq M_{3}^{1 /(q-1)}+M_{4} .
$$

First, we consider $\int_{0}^{1}|y(s)|^{q-1} d s$. By (14), we get

$$
\begin{aligned}
\phi(\lambda) \lambda & \int_{0}^{1} \psi(y(s)) y(s) d s=\phi(\lambda) \int_{0}^{1} x^{(n-1)}(s) y(s) d s \\
= & \phi(\lambda) x^{(n-2)}(1) y(1)-\phi(\lambda) x^{(n-2)}(0) y(0) \\
& \quad-\phi(\lambda) \lambda \int_{0}^{1} x^{(n-2)}(s) f\left(s, x(s), \ldots, x^{(n-2)}(s)\right) d s \\
= & \phi\left(x^{(n-1)}(1)\right)\left(\lambda \frac{\tau}{\gamma} B_{1}\left(\frac{x^{(n-1)}(1)}{\lambda}\right)+\lambda \frac{B}{\gamma}\right) \\
& \quad \phi\left(x^{(n-1)}(0)\right)\left(\lambda \frac{\beta}{\alpha} B_{0}\left(\frac{x^{(n-1)}(0)}{\lambda}\right)+\lambda \frac{A}{\alpha}\right) \\
& -\phi(\lambda) \lambda \int_{0}^{1} x^{(n-2)}(s) f\left(s, x(s), \ldots, x^{(n-2)}(s)\right) d s \\
\leq & \lambda \phi(\lambda) \overline{M_{0}}+\lambda \phi(\lambda) \overline{M_{1}}-\phi(\lambda) \lambda \int_{0}^{1} x^{(n-2)}(s) f\left(s, x(s), \ldots, x^{(n-2)}(s)\right) d s .
\end{aligned}
$$


Then

$$
\begin{aligned}
\int_{0}^{1} \mid & \left.y(s)\right|^{q-1} d s \leq \overline{M_{0}}+\overline{M_{1}} \\
& -\int_{0}^{1} x^{(n-2)}(s) h\left(s, x(s), \ldots, x^{(n-2)}(s)\right) d s-\sum_{i=0}^{n-2} \int_{0}^{1} g_{i}\left(s, x^{(i)}(s)\right) x^{(n-2)}(s) d s \\
\leq & \overline{M_{0}}+\overline{M_{1}}+\sum_{i=0}^{n-2} \int_{0}^{1}\left|g_{i}\left(s, x^{(i)}(s)\right)\right|\left|x^{(n-2)}(s)\right| d s \\
\leq & \overline{M_{0}}+\overline{M_{1}}+\sum_{i=0}^{n-2} g_{\delta, i}\left(\int_{0}^{1}\left|x^{(n-2)}(s)\right|^{m+1} d s\right)^{1 /(m+1)} \\
& +\sum_{i=0}^{n-3} \frac{r_{i}+\epsilon}{[(n-3-i) !]^{m}} \int_{0}^{1}\left|x^{(n-2)}(u)\right|^{m+1} d u+\left(r_{n-2}+\epsilon\right) \int_{0}^{1}\left|x^{(n-2)}(s)\right|^{m+1} d s \\
\leq & \overline{M_{0}}+\overline{M_{1}}+\frac{1}{q} M^{q /(m+1)} \\
& +\psi\left(\sum_{i=0}^{n-2} g_{\delta, i} M^{1 /(m+1)}+\sum_{i=0}^{n-3} \frac{r_{i}+\epsilon}{[(n-3-i) !]^{m}} M+\left(r_{n-2}+\epsilon\right) M\right) \\
= & M_{3} .
\end{aligned}
$$

Then there exists $t_{1} \in[0,1]$ such that $\left|y\left(t_{1}\right)\right| \leq M_{3}^{1 /(q-1)}$. Together with

$$
\begin{aligned}
\left|y^{\prime}(t)\right| & =\left|\lambda f\left(t, x(t), \ldots, x^{(n-2)}(t)\right)\right| \\
& \leq \max _{t \in[0,1],\left|x_{i}\right| \leq M_{2}}\left|f\left(t, x_{0}, x_{1}, \ldots, x_{n-2}\right)\right|=: M_{4},
\end{aligned}
$$

one gets

$$
\|y\|_{\infty}=\max _{t \in[0,1]}|y(t)|=\max _{t \in[0,1]}\left|y\left(t_{1}\right)+\int_{t_{1}}^{t} y^{\prime}(s) d s\right| \leq M_{3}^{1 /(q-1)}+M_{4} .
$$

It follows from (i)-(iii) that

$$
\|(x, y)\| \leq \max \left\{\|x\|,\|y\|_{\infty}\right\} \leq \max \left\{M_{2}, M_{3}^{1 /(q-1)}+M_{4}\right\}=M_{5} .
$$

Thus $\Omega_{0}=\{(x, y) \in D(L): L(x, y)=\lambda N(x, y)$ for $\lambda \in(0,1)\}$ is bounded. This completes Step 1.

SteP 2. Let $\Omega_{1}=\{x \in \operatorname{Ker} L: N x \in \operatorname{Im} L\}$; we prove that $\Omega_{1}$ is bounded. 
Let $x=(0, a) \in \operatorname{Ker} L$ and $N x \in \operatorname{Im} L$. Since

$$
N\left(\begin{array}{l}
0 \\
a
\end{array}\right)(t)=\left(\begin{array}{c}
\psi(a) \\
f(t, 0,0, \ldots, 0) \\
(\beta / \alpha) B_{0}(\psi(a))+(A / \alpha) \\
-(\tau / \gamma) B_{1}(\psi(a))+(B / \gamma)
\end{array}\right)
$$

we deduce from $N x \in \operatorname{Im} L$ and Lemma 3.2 that

$$
\int_{0}^{1} \psi(a) d t=-\frac{\tau}{\gamma} B_{1}(\psi(a))+\frac{B}{\gamma}-\frac{\beta}{\alpha} B_{0}(\psi(a))-\frac{A}{\alpha} .
$$

Then

$$
\frac{\tau}{\gamma} \frac{B_{1}(\psi(a))}{a}+\frac{\beta}{\alpha} \frac{B_{0}(\psi(a))}{a}=\frac{B}{\gamma a}-\frac{A}{\alpha a}-\frac{\psi(a)}{a} .
$$

We prove that there exists a constant $M_{6}>H>0(H$ is given in (A3)) such that $|a| \leq M_{6}$. In fact, one has

$$
\lim _{|a| \rightarrow \infty}\left(\frac{B}{\gamma a}-\frac{A}{\alpha a}-\frac{\psi(a)}{a}\right)=0 \text { or }-\infty .
$$

On the other hand, let $\psi(a)=b$. It follows from (A4) that

$$
\begin{aligned}
\liminf _{|a| \rightarrow+\infty}\left(\frac{\tau}{\gamma} \frac{B_{1}(\psi(a))}{a}+\frac{\beta}{\alpha} \frac{B_{0}(\psi(a))}{a}\right) & =\liminf _{|b| \rightarrow \infty}\left(\frac{\tau}{\gamma} \frac{B_{1}(b)}{\phi(b)}+\frac{\beta}{\alpha} \frac{B_{0}(b)}{\phi(b)}\right) \\
& \geq \frac{\tau}{\gamma} \theta+\frac{\beta}{\alpha} \theta>0 .
\end{aligned}
$$

Then (21) contradicts (22). Hence there exists a constant $M_{6}>H>0$ such that $|a| \leq M_{6}$.

STEP 3. Let

$$
\Omega_{2}=\left\{x \in \operatorname{Ker} L: \lambda \Lambda^{-1} x+(1-\lambda) Q N x=0, \lambda \in[0,1]\right\} .
$$

We prove that $\Omega_{2}$ is bounded.

We will prove that there exists a constant $M_{7}>0$ such that $|a| \leq M_{7}$ for each $(0, a) \in \Omega_{2}$. In fact, let $x=(0, a) \in \Omega_{2}$. One sees that

$$
\lambda(a, 0,0,0)+(1-\lambda)\left(\psi(a)+\frac{\tau}{\gamma} B_{1}(\psi(a))-\frac{B}{\gamma}+\frac{\beta}{\alpha} B_{0}(\psi(a))+\frac{A}{\alpha}, 0,0,0\right)=0 .
$$

Then

$$
a \lambda+(1-\lambda)\left(\psi(a)+\frac{\tau}{\gamma} B_{1}(\psi(a))-\frac{B}{\gamma}+\frac{\beta}{\alpha} B_{0}(\psi(a))+\frac{A}{\alpha}\right)=0 .
$$

If $\lambda=1$, then $a=0$. If $\lambda<1$, then

$$
-\frac{a \lambda}{1-\lambda}+\frac{B}{\gamma}-\frac{A}{\alpha}-\psi(a)=\frac{\tau}{\gamma} B_{1}(\psi(a))+\frac{\beta}{\alpha} B_{0}(\psi(a)) .
$$


It follows that

$$
-\frac{\lambda}{1-\lambda}+\frac{B-A}{a}-\frac{\psi(a)}{a}=\frac{\tau}{\gamma} \frac{B_{1}(\psi(a))}{a}+\frac{\beta}{\alpha} \frac{B_{0}(\psi(a))}{a} .
$$

Since

$$
\limsup _{|a| \rightarrow \infty}\left(-\frac{\lambda}{1-\lambda}+\frac{B-A}{a}-\frac{\psi(a)}{a}\right)<0
$$

and

$$
\liminf _{|a| \rightarrow \infty}\left(\frac{\tau}{\gamma} \frac{B_{1}(\psi(a))}{a}+\frac{\beta}{\alpha} \frac{B_{0}(\psi(a))}{a}\right)>0,
$$

we get a contradiction from $(23)-(25)$. Thus there exists a constant $M_{7}>0$ such that $|a| \leq M_{7}$ for each $(0, a) \in \Omega_{2}$.

STEP 4. Let

$$
\Omega=\left\{x \in X:\|x\|<M_{5}+M_{6}+M_{7}+1\right\} .
$$

Then $\Omega$ is a nonvoid bounded open subset of $X$ centered at zero. It follows from Steps 1-3 that

$$
\Omega \supseteq \Omega_{0} \cup \Omega_{1} \cup \Omega_{2} .
$$

It is easy to see that $L(x, y) \neq \lambda N(x, y)$ for $\lambda \in(0,1]$ and $(x, y) \in D(L) \cap \partial \Omega$; $N(x, y) \notin \operatorname{Im} L$ for every $(x, y) \in \operatorname{Ker} L \cap \partial \Omega$; and $\operatorname{deg}\left(\left.\Lambda Q N\right|_{\operatorname{Ker} L}, \Omega \cap\right.$ Ker $L, 0) \neq 0$. It follows from Lemmas 3.2 and 3.1 that $L(x, y)=N(x, y)$ has at least one solution $(x, y)$ in $\Omega$. Then $x$ is a solution of BVP (5). The proof is complete.

Acknowledgements. The author would like to thank two different anonymous referees for their careful reading of this manuscript and for suggesting some useful changes.

The author is supported by the Science Foundation of Hunan Province (06JJ5008) and by the Natural Sciences Foundation of Guangdong Province (No. 7004569).

\section{References}

[1] R. P. Agarwal, Boundary Value Problems for Higher Order Differential Equations, World Sci., Singapore, 1986.

[2] R. P. Agarwal and F. H. Wong, Existence of positive solutions for non positive higher order BVPs, J. Comput. Appl. Math. 88 (1998), 3-14.

[3] L. Erbe and M. X. Tang, Existence and multiplicity of positive solutions to nonlinear boundary value problems, Differential Equations Dynam. Systems 4 (1996), 313-320.

[4] L. Erbe and H. Y. Wang, On the existence of positive solutions of ordinary differential equations, Proc. Amer. Math. Soc. 120 (1994), 743-748.

[5] R. E. Gaines and J. L. Mawhin, Coincidence Degree and Nonlinear Differential Equations, Lecture Notes in Math. 568, Springer, Berlin, 1977. 
[6] W. C. Lian and F. W. Wong, Existence of positive solutions for higher-order generalized p-Laplacian BVPs, Appl. Math. Lett. 13 (2000), 35-43.

[7] B. Liu and J. S. Yu, Solvability of multi-point boundary value problem at resonance (III), Appl. Math. Comput. 129 (2002), 119-143.

[8] Y. J. Liu, Solutions of Sturm-Liouville boundary value problems for higher-order differential equations, J. Appl. Math. Comput. 24 (2007), 231-243.

[9] S. S. Qi, Multiple positive solutions to BVPs for higher-order nonlinear differential equations in Banach spaces, Acta Math. Appl. Sinica (English Ser.) 17 (2001), 271278.

[10] F. H. Wong, An application of Schauder's fixed point theorem with respect to higher order BVPs, Proc. Amer. Math. Soc. 126 (1998), 2389-2397.

Yuji Liu

Department of Mathematics

Guangdong University of Business Studies

Guangzhou 510000, P.R. China

E-mail: liuyuji888@sohu.com

Received on 4.9.2009;

revised version on 28.10.2010 